\title{
Presenting symptoms and clinical findings in HPV-positive and HPV-negative oropharyngeal cancer patients
}

\section{Carpen, Timo}

2018

Carpen , T , Sjöblom , A , Lundberg , M , Haglund, C , Markkola , A , Syrjänen , S , Tarkkanen , J , Mäkitie , A , Hagström , J \& Mattila , P 2018 , ' Presenting symptoms and clinical findings in HPV-positive and HPV-negative oropharyngeal cancer patients ' , Acta Oto-Laryngologica , vol. 138 , no. 5 , pp. 513-518 . https://doi.org/10.1080/00016489.2017.1405279

http://hdl.handle.net/10138/311877

https://doi.org/10.1080/00016489.2017.1405279

unspecified

acceptedVersion

Downloaded from Helda, University of Helsinki institutional repository.

This is an electronic reprint of the original article.

This reprint may differ from the original in pagination and typographic detail.

Please cite the original version. 


\section{Presenting symptoms and clinical findings in HPV-positive and}

\section{HPV-negative oropharyngeal cancer patients}

Timo Carpén ${ }^{1}$, Anni Sjöblom ${ }^{2}$, Marie Lundberg ${ }^{1}$, Caj Haglund $^{3}$, Antti Markkola ${ }^{4}$, Stina Syrjänen $^{5}$, Jussi Tarkkanen ${ }^{2}$, Antti Mäkitie ${ }^{1,6}$, Jaana Hagström ${ }^{2}$ and Petri Mattila ${ }^{1}$

${ }^{1}$ Department of Otorhinolaryngology - Head and Neck Surgery, University of Helsinki and Helsinki University Hospital, Helsinki, University of Helsinki, Finland

${ }^{2}$ Department of Pathology, Haartman Institute and HUSLAB, University of Helsinki and Helsinki University Hospital, Helsinki, University of Helsinki, Finland

${ }^{3}$ Department of Radiology, University of Helsinki and Helsinki University Hospital, Helsinki, University of Helsinki, Finland

${ }^{4}$ Department of Surgery, University of Helsinki and Helsinki University Hospital, Helsinki, University of Helsinki, Finland

${ }^{5}$ Department of Oral Pathology and Oral Radiology, University of Turku and Turku University Hospital, Turku, University of Turku, Finland

${ }^{6}$ Division of Ear, Nose and Throat Diseases, Department of Clinical Sciences, Intervention and Technology, Karolinska Institutet and Karolinska Hospital, Stockholm, Sweden 
Abstract

Objectives: Oropharyngeal squamous cell carcinoma (OPSCC) is divided in two different disease entities depending on HPV involvement. We investigated differences in presenting symptoms and clinical findings in patients with HPV-positive and negative OPSCC tumors.

Methods: Altogether 118 consecutive patients diagnosed with primary OPSCC between 2012 and 2014 at the Helsinki University Hospital were included. HPV-status of the tumors was assessed by PCR detection of HPV DNA and immunostaining with p16-INK4a antibody.

Results: Fifty-one (47.7\%) of the patients had HPV-positive and 56 (52.3\%) had HPV-negative tumors. Forty-nine $(49 / 51,96.1 \%)$ of the HPV+ tumors were also p16+ showing high concordance. The most common presenting symptom among HPV+/p16+ patients was a neck mass $(53.1 \%)$ whereas any sort of pain in the head and neck area was more frequently related to the HPV-/p16(60.0\%) group. HPV+/p16+ tumors had a tendency to locate in the tonsillar complex and more likely had already spread into regional lymph nodes compared with HPV-/p16- tumors. Smoking and alcohol consumption were significantly more common among HPV-/p16- patients but also rather common among $\mathrm{HPV}+/ \mathrm{p} 16+$ patients.

Conclusion: The present analysis of symptoms and signs confirm that OPSCC can be dichotomized in two distinct disease entities as defined by HPV status.

Keywords: human papillomavirus, oropharynx, p16, symptom, cancer, etiology 


\section{Introduction}

Oropharyngeal squamous cell carcinoma (OPSCC) incidence has been rapidly increasing and HPV infection is considered to be the most significant causal factor responsible for this [1-6]. The majority of HPV-positive head and neck cancers are located in the oropharyngeal region $[1,7]$. HPV infection is an independent risk factor for OPSCC despite tobacco and alcohol use, and nowadays up to $70 \%$ of newly diagnosed OPSCCs are HPV positive $[1,2,6,7]$. Nevertheless, heavy smoking and alcohol consumption are still major risk factors for OPSCC.

Numerous studies have indicated that HPV-positive and HPV-negative OPSCC patients are divided into two different disease groups by different demographic factors, risk profiles and tumor characteristics [1,6,8-10]. Majority of patients affected by HPV-positive OPSCC are found to be younger, generally healthier, sexually active and belong to higher socio-economical group. On the contrary, the HPV-negative OPSCC patients are generally older, have heavy smoking history, and among them profuse alcohol consumption is more common compared with HPV-positive patients. $[9,11]$ The HPV-positive tumors are found to be smaller by T stage, have well-defined borders, and although by the time of diagnosis have more likely spread into regional lymph nodes and nevertheless have more favorable prognosis when compared with HPV-negative tumors $[1,9,10,13-15]$. On the other hand, smoking and alcohol use strongly impair the prognosis even among HPV-positive patients [12].

Analysis and awareness of the symptoms are important as oral and oropharyngeal cancer patients are generally diagnosed relatively late at an advanced stage, which impairs prognosis and survival $[15,16]$. Generally, OPSCCs do not induce symptoms until they are large enough $(>2 \mathrm{~cm}$ or T2) [16] causing local pressure to nearby structures, and at this point they have often already spread into regional lymph nodes $[10,17]$. Diagnosis may be delayed also by patient-dependent factors $[16,18]$. 
Nevertheless, waiting time for diagnosis and treatment has decreased by time in head and neck cancers in general [19].

In an effort to potentially facilitate early diagnosis of OPSCC we wanted to characterize in detail the presenting symptoms and clinical findings of HPV-positive and -negative OPSCC among our patients.

\section{Materials and Methods}

\section{Study population}

The present study is based on a cohort of 118 consecutive patients with newly diagnosed primary OPSCC treated at the Department of Otorhinolaryngology - Head and Neck Surgery at the Helsinki University Hospital during a 2-year time period between February 2012 and February 2014. The study was approved by the Research Ethics Board at the Helsinki University Hospital.

The inclusion criterion was a newly diagnosed OPSCC with no previous primary cancer. Clinical data were collected from electronic medical records. Data included gender, age at diagnosis, comorbidities as defined by the Charlson Comorbidity Index, presenting symptoms, and the time between the start of the presenting symptoms and the time of OPSCC diagnosis. The type of presenting symptoms was categorized in four different types: 1) neck mass, 2) pain in the head and neck area, 3) dysphagia/globus, and 4) other (e.g. bleeding, weight loss and asymptomatic).

History of tobacco smoking was defined as 'never', 'former use', or 'present use'. The magnitude of cigarettes smoked was estimated as years of daily cigarette packages (20 cigarettes) consumed. 
Alcohol use was estimated as 'none', 'moderate drinking', and 'heavy alcohol use'. Heavy alcohol use was estimated as drinking during the past few years more than 24 standard drinks $(12 \mathrm{~g}$ of ethanol) a week for men and more than 16 standard drinks a week for women.

Tumor location was divided into four different categories: 1) tonsil, 2) base of tongue, 3) soft palate, or 4) posterior pharyngeal wall. Tumor spread and stage were evaluated using the TNM classification. Tumor grade was recorded to evaluate the histology.

p16-INK4a immunohistochemistry

We detected the p16-INK4a status by immunohistochemistry (IHC) on paraffin embedded formalin fixed tissue samples. Positive p16 expression was defined when over $70 \%$ of tumor cells were positive. Xylene was used for deparaffinization and rehydration was done in graded series of alcohol. PreTreatment-module (Lab Vision Corp., UK Ltd, UK) was used to treat the tissue slides in Tris-HCl buffer ( $\mathrm{pH} 8.5$ ), and endogenous peroxidase was blocked with $0.3 \%$ Dako REAL Peroxidase-Blocking Solution. Monoclonal mouse anti-human p16-INK (9517 CINtec Histology Kit, MTM laboratories, Germany) was used as primary antibody.

HPV PCR

PCR assay was used for detection of HPV DNA in tissue samples. DNA was extracted from 4-10 um diameter sections cut from paraffin embedded formalin fixed sections and amplified with primer sets 1 and 2 from the Multiplex HPV Genotyping Kit® (DiaMex GmbH, Germany). A negative control contained no genomic DNA to confirm the absence of a contamination in the amplification reactions. HPV genotyping was performed with a Multiplex HPV Genotyping Kit® (DiaMex 
GmbH, Germany) which detects 24 LR- and HR-HPV-genotypes. The labeled hybrids were analyzed with a Luminex LX-100 analyzer (Bio-Plex 200 System, Bio-Rad Laboratories, Hercules, USA).

\section{Statistical analysis}

Data were analyzed with a statistical software program (IBM SPSS Statistics 22, IBM, Somers, IL, USA). The results are presented as mean, range, or as the number of patients when appropriate. The independent samples t-test was used for parametric comparisons, whereas the Mann-Whitney U-test was used with non-parametric data. The Pearson chi-square test was used for frequency data. A two-sided p-value of less than 0.05 was considered statistically significant.

\section{Results}

Altogether, 118 consecutive newly diagnosed oropharyngeal cancer patients were evaluated. Of the patients $89(75.4 \%)$ were male and the mean age was 62 years (range, $41-84)$.

Immunohistochemical p16 status was determined for all primary tumor samples except for one case where the staining could only be made from a lymph node metastasis. This lymph node metastasis was p16 positive and the primary tumor located in the pharyngeal wall. Of the primary tumor samples $80(67.8 \%)$ were p16 positive and $38(32.2 \%)$ were p16 negative.

PCR assay for detection of HPV DNA was done for all except for eleven missing samples (107/118). Fifty-one (51/107, $47.7 \%)$ of the patients were HPV positive and $56(56 / 107,52.3 \%)$ HPV negative. 
All the demographic and clinical parameters were divided into three different subgroups as follows: 1) HPV+/p16+, 2) HPV-/p16- and 3) HPV+/p16-. We compared the two first groups with each other. Furthermore, all p16-positive patients with a known HPV association were compared with p16-/HPVpatients (group 2). Clinical and tumor specific characteristics of p16-positive but HPV-negative patients were close to group 1 patients. Thus, the comparison of HPV-/p16+ group with other subgroups was not relevant. Two patients (2/107) were HPV positive but p16 negative and these are not included in the analyses and tables.

Of the tumors HPV+/p16+ was found in $49(46.7 \%)$ of the 105 tumor samples and $36(33.3 \%)$ patients were HPV-/p16- whereas $21(20.0 \%)$ patients were p16 positive but HPV negative. The concordance of p16 status determining HPV DNA association was $96.1 \%$ (49/51) and specificity was $62.5 \%(35 / 56)$.

Smoking and former smoking classes were significantly more common among HPV-/p16- patients when compared with HPV+/p16+ and with all p16-positive patients. However, smoking was also rather common among HPV+/p16+ cases $(63.3 \%)$. We investigated the HPV-positive cases regarding to their smoking status (HPV+ smokers/ex-smokers vs. HPV+ never smokers) but this aspect had no statistically significant influence on the presenting symptoms or sings (data not shown). Alcohol use in general showed no significant difference between different groups but heavy alcohol use was significantly more common among HPV-/p16- patients when compared with HPV+/p16+ or to p16positive patients. Comorbidities determined by Charlson Comorbidity index showed no differences between different groups.

The median time from onset of symptoms to diagnosis seemed to be slightly lower (2.8 months) among HPV+/p16+ patients compared with HPV-/p16- patients (3.0 months) although the difference was not significant. The clinical characteristics of the 105 OPSCC patients are shown in 
Table 1.

Among HPV+/p16+ patients neck mass was the most common presenting symptom $(26 / 49,53.1 \%)$ and it was statistically significantly (OR 3.82 CI 95\% 1.45 - 10.05) more common compared with HPV-/p16-. Majority of the HPV-/p16- patients (21/35, 60.0\%) indicated any sort of pain in the head and neck area as a presenting symptom. Only seven patients had two presenting symptoms, which could not be separated temporarily. All the results of the presenting symptoms are shown in Table 2 .

The most common tumor location in every subgroup was tonsillar complex and among HPV+/p16+ patients it was significantly more common location $(77.6 \%$, OR 4.61 CI 95\% $1.79-11.89)$ compared with HPV-/p16- group. Among HPV-p16- patients the second most common location was soft palate $(10 / 35,28.6 \%)$ where it located statistically significantly more often $(\mathrm{p}<0.001)$ than in HPV+/p16+ or p16-positive patients. Tumor locations are presented in Table 3.

There was no significant statistical difference in $\mathrm{T}$ stage between different groups. Nodal metastases were more common among HPV+/p16+ patients when compared with HPV-/p16-. N2b was the most common $(26 / 49,53.1 \%) \mathrm{N}$ stage among HPV+/p16+ patients being significantly (OR $3.27 \mathrm{CI}$ 95\% 1.27 - 8.39) more frequent when compared with HPV-/p16-. Distant metastases were present in four cases. The most usual clinical stage was IVa in every group but it was significantly $(\mathrm{p}=$ 0.001) more common among HPV+/p16+ patients $(37 / 49,75.5 \%)$ compared with HPV-p16-. In addition among HPV+/p16+ patients the histological tumor grade was significantly higher compared with HPV-p16- patients. Majority $(43 / 49,87.8 \%)$ of the HPV+/p16+ tumors were of grade III and most $(17 / 34,50.0 \%)$ of the HPV-p16- tumors were of grade II. Regarding both these grades the difference was found to be significant between the groups. Tumor characteristics are shown in Table 4. 


\section{Discussion}

We present a retrospective study on 118 consecutive patients with newly diagnosed oropharyngeal cancer. Our aim was to determine the presenting symptoms and clinical findings of HPV-positive and HPV-negative OPSCC patients in order to define characteristics of these two cancer groups and to aid better detection of OPSCC at an early stage. Our analysis included newly diagnosed OPSCC patients during a two-year follow-up in our referral area of 1.6 million inhabitants covering close to one-third of the entire Finnish population.

HPV status was evaluated by both p16 IHC as well as by HPV PCR of paraffin embedded tissue slices. Immunohistochemical staining of p16 is a sensitive method to screen for HPV in OPSCC and HPV PCR is a rather specific test for HPV involvement. We defined HPV-positive cases as being positive for both p16 IHC and HPV PCR and HPV-negative cases as being negative for both the above assays. Forty-nine (49/51) of the HPV DNA positive patients were also p16 positive showing high concordance $(96.1 \%)$. However, 21 out of the 70 p16-positive cases were HPV-DNA negative. This may indicate that there may be some other mechanisms than HPV-induced mechanisms that lead to p16 up-regulation. On the other hand, during the course of carcinogenesis HPV DNA might be deleted once p16 gets permanently up-regulated. Of note was that the clinical and tumor characteristics of p16-positive but HPV-negative patients and also p16-positive and HPV-positive patients resembled each other suggesting that these tumors may share some aspects of oncogenesis. There were also two cases that were HPV positive but p16 negative. The reasons for this phenomenon remain unclear but possible explanations may include DNA contamination or the presence of HPV DNA that is unrelated to carcinogenesis. Another limitation of our approach was that although p16 IHC was done for all cases $(n=118)$ HPV PCR could only be performed on 107 of the cases. Yet, the number of single positive cases was relatively small and the strength of our approach was that 
defining the HPV status by both the p16 IHC and HPV PCR is a rather reliable method to dissect between HPV-positive and -negative cases.

Neck mass was the most common presenting symptom among HPV-positive patients whereas any sort of pain in the head and neck area was the most often present among HPV-negative patients. A clear majority of the latter patients had a rather nonspecific pain sensation described as sore throat and only a few patients had odynophagia, ear pain, or jaw pain. McIlwain et al. in 2014 [20] showed similar results but the limitation of their study was that the HPV status was only evaluated by p16 IHC. Similarly as in previous studies $[3,6,11]$ the tonsillar complex was the most common tumor location in our study within both groups although it was significantly more usual among HPVpositive when compared with HPV-negative patients. Interestingly, among HPV-/p16- patients soft palate was the second most common tumor location and significantly more common compared with $\mathrm{HPV}+/ \mathrm{p} 16+$ patients. Prior reports $[3,11,12,19,20]$ have shown that $\mathrm{T}$ class is usually lower and $\mathrm{N}$ class higher among HPV-positive patients compared with HPV-negative patients. In the present study T class was lower among HPV-positive patients but the difference compared with HPV negative was not statistically significant probably due to small amount of HPV-negative cases. In our material N class was significantly higher among HPV-positive patients compared with HPV-negative patients, which is in line with earlier reports. In both groups the most common clinical stage was IVa. In conclusion, our results verify the earlier findings $[10,11,20]$ that HPV-positive tumors are smaller by T class and generally present with a neck mass when these have already spread to local lymph nodes whereas HPV-negative tumors are larger by T class and typically expand locally causing only local symptoms such as sore throat.

A clear majority of the patients were male, which supports prior reports $[2,3,6,10,11-13,17,20]$. Some previous studies $[3,9,11,13]$ have shown that HPV-positive patients are more likely to be younger compared with HPV-negative patients but we did not find any clear statistically significant 
age difference between these groups, which is in line with some other earlier reports [10,20]. Tobacco smoking and alcohol consumption were significantly more frequent among HPV-negative patients compared with HPV-positive patients as reported by earlier studies $[3,11,12,17,20]$. However, even two thirds of the HPV-positive patients were current smokers or ex-smokers in the present series which is line in with few other studies [3,12]. In addition, we found no significant difference in the Charlson Comorbidity Index results between these two groups. This points out an interesting observation that HPV-positive OPSCC patients are not always younger, healthier, nonsmokers, and moderate alcohol users as reported by some studies [9,11]. It may indicate that alcohol consumption and tobacco use are rather common among Finnish OPSCC patients regardless of their HPV status. Ang et al. in 2010 showed that OPSCC patients should be divided not just by their HPV status but as well according to tobacco use and especially according to their smoking pack years [12] as prolonged smoking may lead to numerous mutations and extensive tumor heterogeneity, which may make oncologic treatment challenging. We observed that the presenting symptoms of HPV-positive and -negative patients were on average somewhat different reflecting the difference in the pathogenesis of these tumors. Smoking was mainly associated with HPVnegative tumors but additionally many HPV-positive patients were smokers or had a history of smoking. Thus we analyzed whether the smoking status of HPV-positive patients (no smoking history vs. current smoker or previous smoker) would be associated with the type of symptom presented. Our analysis revealed that smoking status within the HPV positive patient group did not statistically significantly stratify the patients according to their symptoms presented. This may indicate that the mutation leading to over-expression of p16 remains the major factor contributing to the oncogenesis of the tumors. We therefore suggest, that more than the signs and symptoms, HPV and p16 status are the relevant factors when determining the best therapeutic option and prognosis for this patient population. 


\section{Conclusion}

The present analysis of symptoms and signs confirm that OPSCC can be dichotomized in two distinct disease entities as defined by HPV status. Tobacco use and alcohol use were rather common among both of our patient groups.

\section{Acknowledgments}

This study was supported by grants from the Helsinki University Hospital research funds.

\section{Disclosure of interest:}

The authors declare no conflict of interest.

\section{References}

[1] Mehanna H., Beech T., Nicholson T. et al. Prevalence of human papillomavirus in oropharyngeal and nonoropharyngeal head and neck cancer - systematic review and meta-analysis of trends by time and region. Head \& Neck 2013;35:747-55.

[2] Lundberg M., Leivo I., Saarilahti K. et al. Increased incidence of oropharyngeal cancer and p16 expression. Acta Otolaryngol 2011;131:1008-11.

[3] Habbous S., Chu KP., Qiu X. et al. The changing incidence of human papillomavirus-associated oropharyngeal cancer using multiple imputation from 2000 to 2010 at a Comprehensive Cancer Centre. Cancer Epidemiol 2013;37:820-9. 
[4] Mork J., Lie AK., Glattre E. et al. Human papillomavirus infection as a risk factor for squamous-cell carcinoma of the head and neck. N Engl J Med 2001;344:1125-1131.

[5] Chaturvedi AK., Engels EA., Pfeiffer RM. et al. Human papillomavirus and rising oropharyngeal cancer incidence in the United States. J Clin Oncol 2011;29:4294-4301.

[6] D’Souza G., Kreimer AR., Viscidi R. et al. Case-control study of human papillomavirus and oropharyngeal cancer. N Engl J Med 2007;356:1944-56.

[7] Ndiaye C., Mena M., Alemany L. et al. HPV DNA, E6/E7 mRNA, and p16INK4a detection in head and neck cancers: a systematic review and meta-analysis. Lancet Oncol 2014;15:1319-31.

[8] Seoane J., Takkouche B., Varela-Centelles P. et al. Impact of delay in diagnosis on survival to head and neck carcinomas: a systematic review with meta-analysis. Clin Otolaryngol 2012;37:99106.

[9] Gillison ML., D'Souza G., Westra W. et al. Distinct risk factor profiles for human papillomavirus type 16-positive and human papillomavirus type 16-negative head and neck cancers. J Natl Cancer Inst 2008;100:407-420.

[10] Cantrell SC., Peck BW., Li G,Wei Q. et al. Differences in imaging characteristics of HPVpositive and HPV-negative oropharyngeal cancers: a blinded matched-pair analysis. AJNR Am J Neuroradiol 2013;34:2005-2009.

[11] Dahlstrom KR., Bell D., Hanby D. et al. Socioeconomic characteristics of patients with 
oropharyngeal carcinoma according to tumor HPV status, patient smoking status, and sexual behavior. Oral Oncol 2015;51:832-8.

[12] Ang KK, Harris J, Wheeler R, Weber R, Rosenthal DI, Nguyen-Tan PF, et al. Human papillomavirus and survival of patients with oropharyngeal cancer. N Engl J 2010;363:24-35.

[13] Fakhry C., Westra WH., Li S. et al. Improved survival of patients with human papillomaviruspositive head and neck squamous cell carcinoma in a prospective clinical trial. J Natl Cancer Inst 2008;100:261-9.

[14] Ragin CCR. \& Taioli E. Survival of squamous cell carcinoma of the head and neck in relation to human papillomavirus infection: review and meta-analysis. Int J Cancer 2007;121:1813-1820.

[15] Gorsky M., Epstein JB., Oakley C. et al. Carcinoma of the tongue: a case series analysis of clinical presentation, risk factors, staging, and outcome. Oral Surg Oral Med Oral Pathol Oral Radiol Endod 2004;98:546-52.

[16] Guggenheimer J., Verbin RS., Johnson JT. et al. Factors delaying the diagnosis of oral and oropharyngeal carcinomas. Cancer 1989;64:932-935.

[17] Psychogios G., Alexiou C., Agaimy A. et al. Epidemiology and survival of HPV-related tonsillar carcinoma. Cancer Med 2014;3:652-9.

[18] Rogers SN., Vedpathak SV. \& Lowe D. Reasons for delayed presentation in oral and oropharyngeal cancer: the patients perspective. Br J Oral Maxillofac Surg 2011;49:349-53. 
[19] Lyhne NM., Christensen A., Alanin MC. et al. Waiting times for diagnosis and treatment of head and neck cancer in Denmark in 2010 compared to 1992 and 2002. Eur J Cancer 2013;9:162733.

[20] McIlwain WR., Sood AJ., Nguyen SA. et al. Initial symptoms in patients with HPV-positive and HPV-negative oropharyngeal cancer. JAMA Otolaryngol Head Neck Surg 2014;140:441-7.

Corresponding author information: Timo Carpén, MD, timo.carpen@ fimnet.fi, Department of Otorhinolaryngology - Head and Neck Surgery, University of Helsinki and Helsinki University Hospital, Kasarmikatu 11-13, 00029 HUS, Helsinki, Finland 